\title{
Profile Scale-Spaces for Multiscale Image Match
}

\author{
Sean $\mathrm{Ho}^{\star}$ and Guido Gerig \\ Department of Computer Science \\ University of North Carolina, Chapel Hill, NC 27599, USA \\ seanho@cs.unc.edu
}

\begin{abstract}
We present a novel statistical image-match model for use in Bayesian segmentation, a multiscale extension of image profile models akin to those in Active Shape Models. A spherical-harmonic based 3D shape representation provides a mapping of the object boundary to the sphere $S^{2}$, and a scale-space for profiles on the sphere defines a scalespace on the object. A key feature is that profiles are not blurred across the object boundary, but only along the boundary. This profile scalespace is sampled in a coarse-to-fine fashion to produce features for the statistical image-match model. A framework for model-building and segmentation has been built, and testing and validation are in progress with a dataset of 70 segmented images of the caudate nucleus.
\end{abstract}

\section{Why Are Anatomical Objects So Hard to Segment?}

Model-based segmentation has come a long way since Kass and Witkin's original snakes [1], but segmentation of anatomical structures from real-world 3D medical images still presents some difficult challenges for automatic methods. Bayesian model-based segmentation balances a geometry prior, guided by a model of the expected object shape, against an image match likelihood, guided by a model of the expected image appearance around the object. Much has been done on the shape representation and prior; here we will focus on the image match model.

In many objects, a simple gradient-magnitude based image-match model is insufficient. The profile of the image across the object boundary can vary significantly from one portion of the boundary to another. Some portions of the boundary might not even have a visible contrast, in which case the shape prior is needed to define the contour. In real-world medical images, the contrast-to-noise ratio is often low, and models need to be robust to image noise.

In our study, one of the applications we focus on is the caudate nucleus in the human brain. From a partnership with our Psychiatry department, we have access to over 70 high-resolution MRIs (T1-weighted, 1x1x1mm) with highquality manual expert segmentations of both left and right caudates. The manual raters, having spent much effort on developing a reliable protocol for manual segmentation, indicate some of the challenges in caudate segmentation, which motivate a multiscale statistical image-match model for automatic methods.

\footnotetext{
^ Supported by NIH-NIBIB P01 EB002779.
} 
Portions of the boundary of the caudate can be localized with standard edge detection at appropriate scales. However, there are also nearby false edges which may be misleading. In addition, where the caudate borders the nucleus accumbens, there is no contrast at the boundary; the manual raters use a combination of shape prior and external landmarks to define the boundary. Figure 1 shows the challenge.
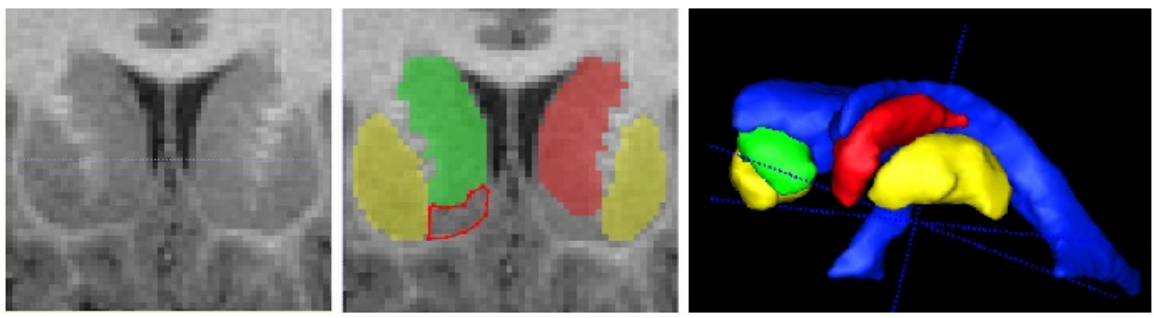

Fig. 1. Coronal slice of the caudate: original T1-weighted MRI (left), and segmented (middle). Right and left caudate are shown shaded in green and red; left and right putamen are sketched in yellow, laterally exterior to the caudates. Nucleus accumbens is sketched in red outline. Note the lack of contrast at the boundary between the caudate and the nucleus accumbens, and the fine-scale cell bridges between the caudate and the putamen. At right is a $3 \mathrm{D}$ view of the caudate and putamen relative to the ventricles.

Another "trouble-spot" for the caudate is where it borders the putamen; there are "fingers" of cell bridges which span the gap between the two. The scale of the object structure relative to the scale of the image noise may swamp single-scale image-match models with the noise.

Many other segmentation tasks in medical images present challenges similar to the caudate; in automatic segmentation methods, this motivates image-match models which are statistically trained and multiscale. This paper focuses on the image match likelihood model, not on the shape prior; the shape prior we use is from the statistical spherical harmonics shape model [2].

\section{Related Work}

There are various image match models in use by current model-based segmentation methods, but relatively few which incorporate scale.

Perhaps the simplest image match model is simply to optimize for high gradient magnitude of the image. This is often used by both classical mesh-based snakes [1] and implicit (geodesic) snakes [3. The gradient magnitude is usually computed at a fixed, global scale. Region-competition snakes use global probability distributions of "inside" intensities vs. "outside" intensities to drive the image match [4]. van Ginneken et al [5] perform a local inside/outside classification using not just the raw greylevels but a high-dimensional $n$-jet feature 
vector, which incorporates multiple scales. Leventon et al [6] train a global profile model that relates intensity values to signed distances from the boundary, incorporating more information than a simple inside/outside classifier, but without treating the scale issue.

Cootes and Taylor's seminal Active Shape Model (ASM) work [7] samples the image along 1D profiles around boundary points, normal to the boundary, using correspondence given by the Point Distribution Model (PDM). Probability distributions are trained for each profile independently. Multi-resolution ASMs [8] use a Gaussian image pyramid in the original image coordinate system. The "hedgehog" model in the 3D spherical harmonic segmentation framework [2] can be seen as a variant of ASMs, and uses a training population linked with correspondence from that geometric model. Profiles have also been used in 2D cardiac MR images [9]. Fenster et al [10] use coarse-scale profiles to summarize sectors of the object boundary.

A different approach is taken by Cootes and Taylor's Active Appearance Models [1], which perform a global Principal Components Analysis on intensities across the whole object after registration. The global PCA is particularly well-suited for capturing global illumination changes in their face recognition applications.

Image features at the boundary may appear at various scales, which motivates a multiscale approach. However, traditional multiscale approaches blur in Euclidean space, which may blur across the object boundary. In the spirit of Canny 12, we wish to construct multiscale features where the blurring is along the boundary and not across the boundary. Our approach is to construct a scale-space on the image profiles, similar to classical scale-spaces 1314 but on a curved non-Euclidean space. We then sample the profile scale-space after the fashion of Laplacian image pyramids [15], to obtain multiscale features upon which Gaussian models are trained using the training population.

\section{Method}

We first describe the process of sampling image profiles across the boundary of the object, in Section 3.1 A scale-space on those profiles is defined in Section 3.2 blurred only along the object boundary, and not across the boundary. Finally, Section 3.3 shows the sampling of the profile scale-space to obtain multiscale features for the statistical model.

\subsection{Extracting Image Profiles of the Object}

The shape representation we use is based on 3D spherical harmonics [2], and provides a diffeomorphic mapping from each object to the unit sphere $S^{2}$. There are many ways to parameterize the objects [16]; our method would work naturally with other parameterizations.

A uniform sampling of the object boundary is obtained from a sampling of the sphere $S^{2}$. At each point on the object boundary, the image is sampled 
evenly along a straight line normal to the boundary, producing an image profile. The first image in Figure 2 shows 512 profiles taken around one caudate from our training set. The 1D ordering of the profiles represents a certain traversal of the 2D surface of the object; adjacent profiles in this visualization are not necessarily adjacent on the surface.

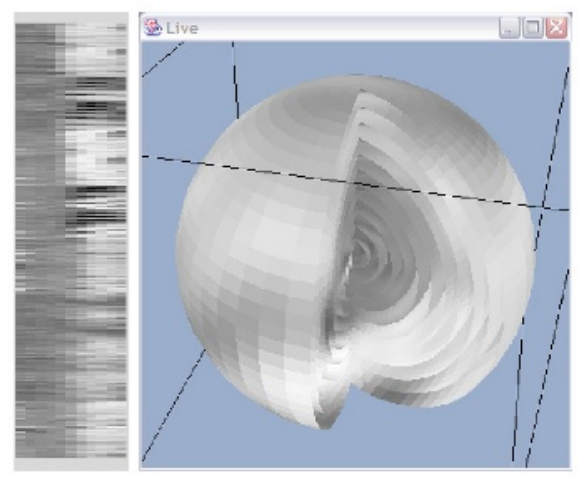

Fig. 2. Intensity profiles from the boundary of a single caudate. At left is a linear visualization of the profiles; the left half is inside the caudate, and the right half is outside. At right is an "onionskin" visualization of the profiles mapped onto concentric spheres. Each sphere represents sampled intensities at a fixed distance away from the boundary, from $-5 \mathrm{~mm}$ to $+5 \mathrm{~mm}$, with $1 \mathrm{~mm}$ spacing.

The right image in Figure 2 shows a different visualization of the same profiles. We use the diffeomorphic mapping of the object to the sphere $S^{2}$ to map each level of the profiles to a sphere. The outermost sphere represents intensities sampled from an "onionskin" $+5 \mathrm{~mm}$ outside the object boundary. Each profile is 11 samples long, so there are 11 concentric spheres in the visualization. The uniform grey matter interior of the caudate can be seen on the interior spheres.

\subsection{Profile Scale-Space}

To extend the classical single-scale profile models to a multiscale framework, we define a scale-space on the profiles sampled on the boundary. The "onionskin" visualization in Figure 2 shows the profiles mapped onto spheres. The intensities at each onionskin level can be mapped onto the sphere $S^{2}$, where a scale-space can be more easily constructed. Each onionskin level is blurred separately, so the blurring stays within each onionskin without blurring across onionskin levels.

The scale-space on $S^{2}$ is defined via the orthonormal spherical harmonic basis on $L^{2}\left(S^{2}\right)$. Note that this is separate from the use of spherical harmonics in the shape representation. Let $f \in L^{2}\left(S^{2}\right)$ be a scalar-valued function on the sphere; e.g. the sampled intensities from a single onionskin about the object. The spherical harmonics $\left\{Y_{l}^{m}\right\}$ form a complete orthonormal basis of $L^{2}\left(S^{2}\right)$, so 
the function $f$ has a Fourier expansion in spherical harmonics:

$$
f(\theta, \phi)=\sum_{l=0}^{\infty} \sum_{m=-l}^{l} c_{l}^{m} Y_{l}^{m}(\theta, \phi),
$$

where $c_{l}^{m}$ are the complex coefficients of the spherical harmonics:

$$
c_{l}^{m}=\int_{\phi=0}^{2 \pi} \int_{\theta=0}^{\pi} f(\theta, \phi) Y_{l}^{m}(\theta, \phi) \sin \theta \mathrm{d} \theta \mathrm{d} \phi .
$$

We make use of the well-known fact that the spherical harmonics are eigenfunctions of the Laplace operator on $S^{2}$, with eigenvalues $l(l+1)$; i.e. $\triangle_{S^{2}} Y_{l}^{m}+l(l+1) Y_{l}^{m}=0$, where the Laplace operator on $S^{2}$ is

$$
\triangle_{S^{2}}=\frac{\partial^{2}}{\partial \theta^{2}}+\frac{\cos \theta}{\sin \theta} \frac{\partial}{\partial \theta}+\frac{1}{\sin ^{2} \theta} \frac{\partial^{2}}{\partial \phi^{2}}
$$

We define the scale-space of $f$ on $S^{2}$ to be $\Phi(f): S^{2} \times R^{+} \rightarrow R$ given by:

$$
\Phi(f)(\theta, \phi ; \sigma)=\sum_{l=0}^{\infty} \sum_{m=-l}^{l} e^{-l(l+1) \sigma} c_{l}^{m} Y_{l}^{m}(\theta, \phi) .
$$

This is the solution to the diffusion equation, $\frac{\partial \Phi(f)}{\partial \sigma}=\triangle_{S^{2}} \Phi(f)$.

The scale-space on the sphere defines a scale-space on the image profiles, via the diffeomorphic mapping provided by the shape representation. If the diffeomorphism were an isometry, the Laplacian on $S^{2}$ would map to the LaplaceBeltrami operator on the surface of the object, and our scale-space would be equivalent to Laplace-Beltrami blurring on the object surface. In general, such an isometry is impossible to obtain, but we have an approximation, since the mapping provided by the spherical harmonic shape representation preserves area exactly and approximately preserves angles. The scale-space on image profiles is constructed using a spherical harmonic basis for the image intensities on each "onionskin", analogous to the use of the spherical harmonic basis for the $(x, y, z)$ coordinates of the boundary.

\subsection{Multiscale Features and Statistical Model}

With a scale-space defined on the image profiles about the object boundary, we sample features from the scale-space in a coarse-to-fine fashion, and build a statistical model on the multiscale tree of features. The coarse-to-fine sampling follows the recursive triangular subdivision of the sphere. In our implementation, we use 512 samples at the finest $\sigma$ scale, down to 8 samples at the coarsest scale, with a total of four scale levels. A schematic of the sampling scheme is illustrated in the left image in Figure 3 The node at the top of the tree represents a single coarse-scale profile summarizing an eighth of the boundary. 


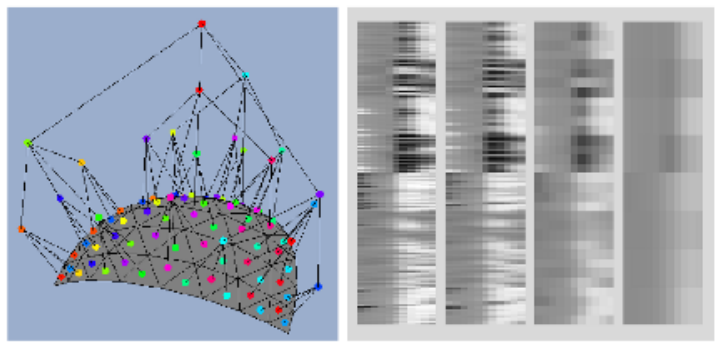

Fig. 3. At left, a visualization of the coarse-to-fine sampling of the profile scale-space. Distance away from the grey sphere indicates coarser scale in the scale-space. At right, average profiles across the training population, at four scales, fine to coarse.

These blurred profiles are calculated for each object in the training population. The right image in Figure 3 shows the mean profiles at multiple scales, where the mean is taken at corresponding locations over the training population. Correspondence is established via the spherical harmonic parametrization [2]. Again, in this visualization, the ordering of the profiles from top to bottom represents a traversal of the object boundary; this blurring is not the same as a simple $y$-axis blur.

Finally, the features used to build the statistical model are scale residuals: differences between each profile at scale (each node in the tree in Figure 31) and its parent profile (parent in the tree, at coarser scale). The eight profiles at the coarsest scale are included unchanged as features. This is analogous to the classical Laplacian image pyramid [15] in Euclidean space. Each scale-residual profile is then modelled with a Gaussian. With profiles 11 samples long, we have $(512+128+32+8)$ Gaussian models, each 11-dimensional. The local Mahalanobis distances are summed to produce a global goodness-of-fit. This model is similar to the single-scale profile model in Active Shape Models, which would have 512 Gaussians, each 11-dimensional, and in contrast with Active Appearance Models, which would have a single very high dimensional Gaussian.

\section{Ongoing/Future Work}

\subsection{Segmentation}

We have implemented a self-contained framework in Mathematica for building this model and using it for segmentation. The shape representation and prior is from Brechbühler and Kelemen, et al 22. The Bayesian segmentation is just an optimization of the posterior, the product of the shape prior and the image-match likelihood. We use Mathematica's built-in NMinimize non-linear optimization routines. The dimension of the search space is only 18; we search over the 12 main eigenmodes of shape variation, and let translation and rotation be unconstrained. Test segmentations, initialized at the mean shape and pose, have been run on a couple test images, with promising results. 

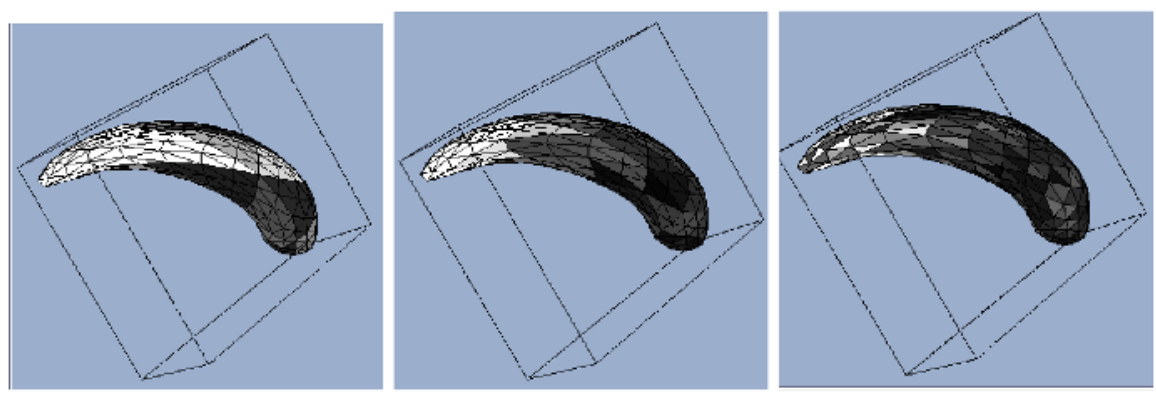

Fig. 4. Local image match goodness-of-fit textured onto deformable caudate segmentation, at initialization (after 1 iteration). White represents worse fit, dark represents better fit. The image match is computed at multiple scales; three scales are shown from coarse to fine.

\subsection{Validation}

Validation is in progress on the new image match model, as well as its use in segmentation. It is anticipated that due to increased robustness to noise and better modelling of population variability, our new multiscale profile model will yield segmentations closer to the manual expert than those from the single-scale profile model. We have access to a large database of over 70 segmented images, as well as intra- and inter-rater reliability figures for the manual raters. Evaluation of specificity/compactness and generalizability of the profile model itself is also planned.

\section{Conclusions}

We present a new multiscale statistical image profile model for use in Bayesian segmentation. The complex image appearance of anatomical structures in noisy medical images motivates a multiscale approach to modelling image profiles. We make use of the spherical harmonic shape representation to map the boundary profiles onto the sphere, where we define a scale-space which blurs image intensities along the object boundary, but not across the boundary. From the continuous scale-space, profiles are sampled at various locations and scales, and scale residuals akin to the Laplacian image pyramid are used as features in the statistical model. We have built the model and run preliminary segmentation tests; in-depth validation is in progress.

Acknowledgments. The caudate images and expert manual segmentations are funded by NIH RO1 MH61696 and NIMH MH 64580 (PI: Joe Piven). Manual segmentations are by Michael Graves, with protocol development by Rachel Gimpel and Heather Cody Hazlett. Image processing, including Figure 1 by Rachel Gimpel. The formalism for a scale-space on $S^{2}$ arose from discussions with Remco Duits at the Technical University of Eindhoven. 


\section{References}

1. M. Kass, A. Witkin, and D. Terzopoulos, "Snakes: Active shape models," International Journal of Computer Vision, vol. 1, pp. 321-331, 1987.

2. András Kelemen, Gábor Székely, and Guido Gerig, "Elastic model-based segmentation of $3 \mathrm{~d}$ neuroradiological data sets," IEEE Transactions on Medical Imaging (TMI), vol. 18, pp. 828-839, October 1999.

3. H. Tek and B.B. Kimia, "Volumetric segmentation of medical images by threedimensional bubbles," Computer Vision and Image Understanding (CVIU), vol. 65, no. 2, pp. 246-258, 1997.

4. S. Zhu and A. Yuille, "Region competition: Unifying snakes, region growing, and Bayes/MDL for multi-band image segmentation," in International Conference on Computer Vision (ICCV), 1995, pp. 416-423.

5. Bram van Ginneken, Alejandro F. Frangi, Joes J. Staal, Bart M. ter Haar Romeny, and Max A. Viergever, "A non-linear gray-level appearance model improves active shape model segmentation," in Proc. of IEEE Workshop on Mathematical Methods in Biomedical Image Analysis (MMBIA) 2001, 2001.

6. M. Leventon, O. Faugeraus, and W. Grimson, "Level set based segmentation with intensity and curvature priors," in Workshop on Mathematical Methods in Biomedical Image Analysis Proceedings (MMBIA), June 2000, pp. 4-11.

7. Timothy F. Cootes, A. Hill, Christopher J. Taylor, and J. Haslam, "The use of active shape models for locating structures in medical images," in Information Processing in Medical Imaging (IPMI), 1993, pp. 33-47.

8. T. Cootes, C. Taylor, and A. Lanitis, "Active shape models: Evaluation of a multiresolution method for improving image search," 1994.

9. Nicolae Duta, Anil K. Jain, and Marie-Pierre Dubuisson-Jolly, "Learning-based object detection in cardiac MR images," in International Conference on Computer Vision (ICCV), 1999, pp. 1210-1216.

10. Samuel D. Fenster and John R. Kender, "Sectored snakes: Evaluating learnedenergy segmentations," IEEE Transactions on Pattern Analysis and Machine Intelligence, vol. 23, no. 9, pp. 1028-1034, 2001.

11. Timothy F. Cootes, Gareth J. Edwards, and Christopher J. Taylor, "Active appearance models," IEEE Transactions on Pattern Analysis and Machine Intelligence (PAMI), vol. 23, no. 6, pp. 681-685, 2001.

12. J.F. Canny, "A computational approach to edge detection," IEEE Trans on Pattern Analysis and Machine Intelligence (PAMI), vol. 8, no. 6, pp. 679-697, November 1986.

13. J. J. Koenderink, "The structure of images," Biological Cybernetics, vol. 50, pp. 363-370, 1984.

14. L. M. J. Florack, B. M. ter Haar Romeny, J. J. Koenderink, and M. A. Viergever, "The Gaussian scale-space paradigm and the multiscale local jet," International Journal of Computer Vision (IJCV), vol. 18, no. 1, pp. 61-75, April 1996.

15. Peter J. Burt and Edward H. Adelson, "The laplacian pyramid as a compact image code," IEEE Transactions on Communications, vol. COM-31,4, pp. 532-540, 1983.

16. Martin A. Styner, Kumar T. Rajamani, Kutz-Peter Nolte, Gabriel Zsemlye, Gabor Szekely, Chris J. Taylor, and Rhodri H. Davies, "Evaluation of 3d correspondence methods for model building," in Information Processing in Medical Imaging (IPMI), 2003. 